\title{
Representation of Locally Convex Algebras
}

\author{
L. OUBBI
}

\begin{abstract}
We deal with the representation of locally convex algebras. On one hand as "subalgebras" of some weighted space $C V(X)$ and on the other hand, in the case of uniformly $A$-convex algebras, as inductive limits of Banach algebras. We also study some questions on the spectrum of a locally convex algebra.
\end{abstract}

\section{INTRODUCTION}

A locally convex algebra is an algebra together with a Hausdorff locally convex topology such that the multiplication of $E$ is separately continuous. We denote by $M$ (resp. $M^{\#}$ ) the assumed nonvoid set of all continuous (resp. algebraic) characters of $E$. Endowed with the weak topology generated by $E, M$ and $M^{\#}$ are Hausdorff completely regular spaces. $M$ will be called the spectrum of $E$ and $M^{\#}$ its algebraic spectrum. This work consists of three independant sections. In [9], conditions are given under which $E$ is embedded algebraically (and topologically) in some $C(X)$ with the compact open topology or a weaker topology. A.C. Cochran shows in [4] that every semisimple uniformly $A$-convex algebra $E$ can be embedded continuously, via the Gelfand map

1991 Mathematics Subject Classification: $46 \mathrm{H} 15,46 \mathrm{H20}, 46 \mathrm{E25}$

Editorial Complutense. Madrid, 1994. 
$G$, in some subspace $H$ of $C(M)$ with a weighted topology. The hypothesis of the semisimplicity is not enough in Cochran's result. Contrarily to an affirmation of Cochran, there are semisimple uniformly $A$-convex algebras wich are not strongly semisimple. In fact if $E$ is the algebra $C^{b}(\mathbf{R})$ with the topology defined by the seminorms $\left(I_{n}\right)_{n \geq 0}$, where $I_{n}(f)=\int_{-n}^{n}|f(x)| d x$ if $n>0$ and $I_{0}(f)=\sup _{x \in[0,1]}|f(x)|, f \in E$, then $E$ is a semisimple uniformly $A$-convex algebra whose spectrum $M$ is homeomorphic to $[0,1]$, hence $E$ is not strongly semisimple, and it is easily seen that $E$ can not be embedded even algebraically in $C[0,1]$. We give, in the first part of this work, a correct statement and an improvement of Cochran's result. We show that every strongly semisimple locally convex algebra (l.c.a.) $E$ can indeed be embedded continuously (as a locally convex algebra) in some weighted space $C V(X)(C V(X)$ need not be itself a l.c.a.), and, with some additional conditions, that $E$ is even dense in $C V_{0}(X)$. The second section gives a representation of locally complete uniformly $A$-convex algebras as inductive limits of $\mathrm{Ba}$ nach algebras. This sharpens a result of [11], where the author assumes that $E$ is sequentially complete and has an identity, 'and describes very well the structure of uniformly $A$-convex algebras. The last section is devoted to some questions on the spectrum $M$ and its behaviour with respect to $M^{\#}$. We show, for example, that if $E$ has an identity such that an element $x$ in $E$ is invertible whenever $x$ does not belong to the kernel of any continuous character, then $M$ is dense in $M^{\#}$. In such a situation, $M$ is equibounded if and only if $M^{\#}$ is equibounded.

\section{REPRESENTATION OF LOCALEY CONVEX ALGEBRAS}

A locally convex algebra $E$ is said to be strongly semisimple if $x=0$ whenever $\chi(x)=0$ for every $\chi \in M$. Such an algebra is necessarily commutative and Hausdorff. We will denote by $G$ the Gelfand map of $E$. It is defined from $E$ into $C(M)$ by $G(x):=\widehat{x}$ with $\widehat{x}(f)=f(x), f \in M$. It is clear that $G$ is one to one if and only if $E$ is strongly semisimple. If $\tau_{e}$ denotes the topology of uniform convergence on the equicontinuous subsets of $M, G$ is continuous into $\left(C(M), \tau_{e}\right)$ [8]. Moreover [9], $G$ is open into $C(M)$ with the compact open topology if and only if every equicontinuous subset $H$ of the topological dual $E^{\prime}$ of $E$ is contained in 
a multiple of the closed convex hull of some equicontinuous subset of $M$. In particular, $E$ must be $m$-convex.

Now, let $X$ be a Hausdorff completely regular space. A nonnegative upper semi-continuous function on $X$ is called a weight. A family of weights is called a Nachbin family if:

1. For every $x \in X$, there is $v \in V$ such that $v(x) \neq 0$.

2. For any $v_{1}, v_{2} \in V$ and $\tau>0$, there is $v \in V$ such that $\max \left(r v_{1}, r v_{2}\right) \leq$ $v$.

We will consider the following weighted spaces:

$C V(X):=\{f \in C(X):$ for every $v \in V, v f$ is bounded $\}$, $C V_{0}(X):=\{f \in C(X):$ for every $v \in V$, $v f$ vanishes at infinity $\}$.

Endowed both with the weighted topology $\tau_{\omega}$ defined by the seminorms

$$
P_{v}(f):=\sup \{v(x)|f(x)|, x \in X\}, v \in V,
$$

$C V_{0}(X)$ is a closed subspace of $C V(X)$. Moreover, if we put $W:=$ $V C_{0}^{+}$, where $C_{0}^{+}$is the set of all nonnegative functions on $X$ vanishing at infinity, we get a Nachbin family such that $C V(X)$ is continuously embedded in $C W_{0}(X)$.

If $E$ is a commutative Hausdorff locally convex algebra, then its spectrum $M$ is a Hausdorff completely regular space. Using an idea of [4], we get

1. Proposition. If $E$ is strongly semisimple, then there exists a Nachbin family on $M$ such that $E$ is continuously embedded, via $G$, in $C V(M)$. Moreover, $\left(G(E), \tau_{\omega}\right)$ is a locally convex algebra.

Proof. Let $\left(P_{\lambda}\right)_{\lambda \in \Gamma}$ be a family of seminorms defining the topology of $E$. Set, for every $\lambda \in \Gamma$ and every $\chi \in M$ :

$$
\mu_{\lambda}(\chi)= \begin{cases}\|\chi\|_{B_{\lambda}^{0}}:=\sup \{|\chi(x)|, & \left.P_{\lambda}(x) \leq 1\right\} \text { if } \chi \in E_{B_{\lambda}^{0}}^{\prime} \\ +\infty & \text { otherwise }\end{cases}
$$


where $B_{\lambda}^{0}$ is the polar of the unit pseudo-ball $B_{\lambda}$ of $P_{\lambda}$ and $E_{B_{\lambda}^{0}}^{\prime}$ its linear hull. Then $\mu_{\lambda}$ is a lower semi-continuous function on $M$ and $v_{\lambda}:=\mu_{\lambda}^{-1}$ (with $\frac{1}{+\infty}=0$ ) is a weight. Put $V:=\left\{r v_{\lambda}: r>0\right.$ and $\lambda \in \Gamma\}$. This is a Nachbin family. In fact, if $\chi \in M$, then there is $\lambda \in \Gamma$ and $c>0$ such that $|\chi(x)| \leq c P_{\lambda}(x), \forall x \in E$. Therefore $\chi \in E_{B_{\lambda}^{o}}^{\prime}$ and then $\mu_{\lambda}(\chi)<+\infty$. Hence $v_{\lambda}(\chi) \neq 0$. Moreover, if $P_{\lambda} \leq P_{\lambda^{\prime}}$, then $v_{\lambda} \leq v_{\lambda^{\prime}}$. Consider now the space $C V(M)$ with the weighted topology $\tau_{\omega}$. If $x \in E, \lambda \in \Gamma$ and $r>0$ satisfy $x \in r B_{\lambda}$, then:

$$
\begin{aligned}
P_{u \lambda}(\widehat{x}) & =\sup _{x \in M_{\lambda}} \frac{|\chi(x)|}{\sup _{y \in B_{\lambda}}|\chi(y)|} \\
& \leq \sup _{x \in M_{\lambda}} \frac{|\chi(x)|}{\mid \overline{\chi\left(\frac{x}{r}\right) \mid}=r}
\end{aligned}
$$

where $M_{\lambda}:=M \cap E_{B_{i}^{0}}^{\prime}$. Hence $G(E) \subset C V(M)$ and for every $x \in E$ and $\lambda \in \Gamma, P_{v_{\lambda}}(\widehat{x}) \leq P_{\lambda}(x)$, whence the continuity of $G$. To show that $\left(G(E), \tau_{\omega}\right)$ is a locally convex algebra, it suffices to show that for every $x \in E$ and $\lambda \in \Gamma$, there are $c>0$ and $\lambda^{\prime}$ with $v_{\lambda}|\widehat{x}| \leq c v_{\lambda^{\prime}}$. But if for every $y \in E, P_{\lambda}(x y) \leq r P_{\lambda^{\prime}}(y)$, then $\frac{1}{r} x B_{\lambda^{\prime}} \subset B_{\lambda}$ and for every $\chi \in M$, we have:

If $\chi \notin M_{\lambda}, v_{\lambda}(\chi)=0$ and then $v_{\lambda}|\widehat{x}| \leq c v_{\alpha}$ for every $\alpha \in \Gamma$ and $c>0$. Now if $\chi \in M_{\lambda}$,

$$
\begin{aligned}
v_{\lambda}(\chi)|\chi(x)| & =\frac{|\chi(x)|}{\sup _{y \in B_{\lambda}}|\chi(y)|} \\
& \leq \frac{|\chi(x)|}{\sup _{y \in B_{\lambda^{\prime}}}\left|\chi\left(\frac{1}{r} x y\right)\right|} \\
& \leq r \frac{1}{\sup _{y \in B_{\lambda^{\prime}}}|\chi(y)|}=r v_{\lambda^{\prime}}(\chi) .
\end{aligned}
$$

Remark. If $E$ has an identity, $C V(M)$ contains the constant function 1 . then the weights $v_{\lambda}$ are bounded on $M$. Actually, they even 
vanish at infinity. Indeed, the set $\left\{\chi \in M: v_{\lambda}(\chi) \geq \epsilon\right\}$ is exactly $M \cap\left(\epsilon B_{\lambda}\right)^{0}$. Since $M$ is closed in $E^{\prime}$, the result is a consequence of the Banach-Alaoglu theorem.

$M_{\lambda}$ in the proof of 1 . is exactly the set of all $P_{\lambda}$-continuous characters. It is also the set where $v_{\lambda}$ does not vanish. If $M_{\lambda}$ is $P_{\lambda^{-}}$ equicontinuous (in particular if $E$ is $m$-convex), then it is equal to the support supp $v_{\lambda}$ of $v_{\lambda}$ and $\inf \left\{v_{\lambda}(\chi), \chi \in M_{\lambda}\right\} \neq 0$, hence the weighted topology $\tau_{\omega}$ is stronger than the topology of uniform convergence on the $M_{\lambda^{\prime}} s$. If, in addition, $E$ has an identity, these topologies coincide.

2. Proposition. If $\left(E,\left(P_{\lambda}\right)_{\lambda \in \Gamma}\right)$ is a strongly semisimple l.c.a. with identity such that every $M_{\lambda}$ is $P_{\lambda}$-equicontinuous, then $E$ is continuously embedded in $C V_{0}(M)$. If, in addition, $E$ has an involution * such that $\chi\left(x^{\star}\right)=\overline{\chi(x)}$, for every $x \in E$ and $\chi \in M$, then $G(E)$ is dense in $C V_{0}(M)$.

Proof. Let $x \in E, \epsilon>0$ and $\lambda \in \Gamma$ be given. If $v_{\lambda}(\chi)|\chi(x)| \geq \epsilon$, then

$$
|\chi(x)| \geq \epsilon \mu_{\lambda}(\chi)=\epsilon \sup _{y \in B_{\lambda}}|\chi(y)|
$$

and $\chi$ is necessarily in $M_{\lambda}$. Moreover, there is a real number $c$ such that $|\psi(y)| \leq c$ for all $\psi \in M_{\lambda}$ and $y \in B_{\lambda}$. Then $M_{\lambda}$ is compact and so is the set

$$
N(\lambda, x):=\left\{\psi \in M: v_{\lambda}(\psi)|\psi(x)| \geq \epsilon\right\} .
$$

Therefore $\widehat{x}$ belongs to $C V_{0}(M)$. The density of $G(E)$ derives from a classical density theorem for $C V_{0}(X)$ (cf. [12], Theorem 3.2 page 284).

By considering the Nachbin family $W=V C_{0}^{+}$, one can show that if $E$ is strongly semisimple with identity and has an involution * such that $\chi\left(x^{\star}\right)=\overline{\chi(x)}$ for every $\chi \in M$ and $x \in E$, then $E$ is dense in $C W_{0}(M)$ and the embedding map is continuous.

3. Proposition. Let $E$ be strongly semisimple such that for every $\lambda \in \Gamma$, there is $\lambda^{\prime} \in \Gamma$ and $c>0$ so that for every $x \in E$ there is $\chi \in M_{\lambda^{\prime}}$ with

$$
P_{\lambda}(x)|\chi(y)| \leq c|\chi(x)| P_{\lambda^{\prime}}(y), y \in E .
$$


Then $E$ can be algebraically and topologically embedded in $C V(M)$.

Proof. We only have to show that $G^{-1}$ is continuous. Let $\lambda \in \Gamma$ be given. There is $\lambda^{\prime}$ and $c>0$ such that:

$\forall x \in E, \exists \chi \in M_{\lambda^{\prime}}: P_{\lambda}(x)|\chi(y)| \leq c|\chi(x)| P_{\lambda^{\prime}}(y), \forall y \in E$

Then

$$
P_{\lambda}(x)|\chi(y)| \leq c|\chi(x)|, \forall y \in B_{\lambda^{\prime}}
$$

Hence $P_{\lambda}(x) \leq c \frac{|\chi(x)|}{\sup _{y \in B_{\lambda^{\prime}}^{\prime}}|\chi(y)|} \leq c P_{v_{\lambda}^{\prime}}(\widehat{x})$ and we are done.

\section{CASE OF UNIFORMLY A-CONVEX ALGEBRAS}

In this section, we give a representation result in the case of uniformly $A$-convex algebras. We describe them as inductive limits of $\mathrm{Ba}$ nach algebras. M. Oudades [11] has shown, with a relatively complicated method, that every sequentially complete unital uniformly $A$-convex algebra $E$ is a bornological inductive limit of Banach algebras. This means that there is an inductive system $E_{i}$ of Banach algebras whose inductive limit (as an algebra) is $E$ and that a subset of $E$ is bounded if and only if it is contained and bounded in some $E_{i}$. We give here a very easy proof of this result. In fact, we show even more. We do not need an identity, nor sequential completeness. The local completeness is sufficient for our proof. Recall that a subset $V$ of an algebra $E$ is said to be $A$-convex if it is absolutely convex and absorbes $x V$ and $V x$ for every $x \in V$. If $V$ is, in addition, idempotent, then it is called m-convex. An $A$-bounded [5] set is a bounded set which is absorbed by a bounded $A$-convex set. An $m$-bounded [13] set is defined in the same way. Every $m$-bounded set is $A$-bounded, but the converse is not true even in a locally $\mathrm{m}$-convex algebra contrarily to an assertion of [5]. In fact, if $E$ is the algebra $C_{c}^{b}(\mathbf{R})$ of all continuous and bounded functions on $\mathbf{R}$ with the compact open topology, then the set $B:=\{f \in E:|f(x)| \leq|x|, x \in \mathbf{R}\}$ is $A$-bounded but not m-bounded.

If $V \subset E$ is $A$-convex, we denote by $V^{\prime}$ the set $\{x \in V: x V \subset V\}$. This is an $m$-convex set generating the same linear space as $V$. If $V$ is 
closed, so is also $V^{\prime}$. The corner-stone in the sequel is the following easy observation.

1. Lemma. In a uniformly A-convex algebra, every bounded set is A-bounded.

Proof. Let $(E, \mathbf{P})$ be a uniformly $A$-convex algebra. For every $\left(\alpha_{P}\right)_{P \in \mathbf{P}} \in\left(\mathbf{R}_{+} \backslash\{0\}\right)^{\mathbf{P}}$, put $B(\alpha):=\left\{x \in E: P(x) \leq \alpha_{P}\right\}$. This is a bounded set, and the collection of such sets in a base for the bounded sets of $E$. but if $P(x y) \leq c P(y)$ and $P(y x) \leq c P(y)$ for all $y \in E$ and all $P \in \mathcal{P}$, then $x B_{\alpha} \cup B_{\alpha} x \subset c B_{\alpha}$. Hence $B_{\alpha}$ is $A$-convex.

2. Proposition. 1. Every Hausdorff uniformly A-convex algebra $E$ is bornologically the inductive limit of an inductive system $\left(E_{\alpha}\right)_{\alpha}$ of $A$-normed algebras. If, in addition, $E$ is locally complete, the $E_{\alpha^{\prime}} s$ can be chosen to be Banach algebras.

2. If $E$ is bornological as a locally convex space, the same statement as in 1: holds with "bornologically" replaced by "topologically".

3. If $E$ is pseudo-complete, it is a directed union of Banach algebras with continuous injections.

Proof. With Lemma 2.1. in mind, the proof of 1 . and 2. is standard. Note just that for any closed bounded $A$-convex set $B$ the linear hull $E_{B}$ of $B$ endowed with the gauge of $B$ is an $A$-normed algebra, and if $E$ is locally complete, then the gauges of $B$ and of $B^{\prime}:=\{x \in B$ : $x B \subset B\}$ are equivalent Banach algebra norms on $E_{B}$.

3. If $E$ is only pseudo-complete, it is not clear that the gauges of $B$ and $B^{\prime}$ are equivalent and we do not know wether the family $\left(E_{B_{i}^{\prime}}\right)_{i}$ is a topological inductive systems, where $\left(B_{i}\right)_{i}$ is a fundamental base for the bounded sets in $E$ consisting of closed $A$-convex sets. However $E$ is the union of the upward directed family $\left(E_{B_{i}^{\prime}}\right)_{i}$ of (Banach) algebras, and since each $B^{\prime}$ is bounded, the injections are continuous.

Most of the good properties of uniformly A-convex algebras can be deduced from this proposition. In particular, every element of such an algebra is regular (bounded of [2]), the spectrum of an element of a pseudo-complete uniformly $A$-convex algebra $E$ is compact, and if $E$ is locally complete, it is a pseudo-Banach algebra in the sense of [3] or a 
complete b:m.c.a. in the sense of [1] and [6]. The spectrum of such an algebra is equibounded...

\section{SPECTRUM OF A LOCALLY CONVEX ALGEBRA}

If $E$ is a commutative Hausdorff locally convex algebra, one can ask: When is $M$ dense in $M \#$ ? This question is suggested by the following (counter-) examples:

1. Let $E$ be the algebra $C[0,1]$ of all continuous functions on $[0,1]$ equipped with the topology of the norm of $L^{1}[0,1]$. This is a commutative Hausdorff 1.c.a. (in fact an $A$-normed algebra) with identity such that $M=\phi$ and $M^{\#}=[0,1]$. Hence $M$ is not dense in $M^{\#}$.

2. If $E$ is the algebra $H(\mathbf{C})$ of all holomorphic functions on $\mathbf{C}$ equipped with the topology of uniform convergence on the closed unit disk $D$, then $E$ is a commutative strongly semisimple normed algebra such that $M$ is not dense in $M^{\#}$.

The l.c.a. $E$ is said to have property $(W)$ if i) $E$ has an identity and an element $x$ in $E$ is invertible whenever $x$ does not belong to the kernel of any continuous character or ii) $E$ has no identity, $0 \in \bar{M}$, and $x \in E$ is quasi-invertible whenever $\chi(x) \neq 1$ for every $\chi \in M$.

1. Proposition. Let $E$ be a commutative Hausdorff l.c.a. with property $(W)$. Then, in the following cases, $M$ is dense in $M^{\#}$ :

1. $G(E)$ is a $C^{b}(M)$-module.

2. $\mathbf{K}=\mathbf{C}$ and $E$ has an involution * such that for every $\chi \in M$ and $x \in E, \chi\left(x^{\star}\right)=\overline{\chi(x)}$.

3. $\mathbf{K}=\mathbf{R}$.

Proof. Let $\chi_{0} \in M$ and $\epsilon>0$ be given. We have to show that for arbitrary $x_{1}, x_{2}, \ldots, x_{n} \in E, V\left(\chi_{0}, x_{1}, x_{2}, \ldots, x_{n}, \epsilon\right):=\{\chi \in M$ : $\left.\left|\chi\left(x_{i}\right)-\chi_{0}\left(x_{i}\right)\right|<\epsilon, i=1,2, \ldots, n\right\}$ is nonvoid. Assume first that $n=1$ and that for $x \in E$ and $\epsilon>0, V(\chi 0, x, \epsilon)=\phi$. Then $\left|\chi(x)-\chi_{0}(x)\right| \geq \epsilon$.

Case 1: $E$ has an identity. 
If $\chi_{0}(x)=0$, then for every $\chi \in M, \chi(x) \neq 0$ and then $x$ is invertible. $1=\chi_{0}\left(x x^{-1}\right)=\chi_{0}(x) \chi_{0}\left(x^{-1}\right)=0$ is absurd.

If $\chi_{0}(x) \neq 0$, then due to property $(W), y:=x-\chi_{0}(x) 1$ must be invertible. But this leads to the same absurdity.

Case 2: $E$ has no identity.

The fact $\chi_{0}(x)=0$ contradicts property $(W)$. Suppose then $\chi_{0}(x) \neq 0$. Hence $\chi\left(\frac{x}{\chi_{0}(x)}\right) \neq 1, \forall \chi \in M$. Thus $\frac{x}{\chi_{0}(x)}$ is quasi-invertible, which is impossible.

Consiser now $x_{1}, x_{2}, \ldots, x_{n}$ in $E, \epsilon>0$ and set:

$$
\begin{aligned}
V^{\#}\left(\chi_{0}, x_{1}, x_{2}, \ldots, x_{n}, \epsilon\right) & :=\left\{\chi \in M^{\#}:\left|\chi\left(x_{i}\right)-\chi_{0}\left(x_{i}\right)\right|<\epsilon,\right. \\
i & =1,2, \ldots, n\} .
\end{aligned}
$$

This is an open neighbourhood of $\chi_{0}$ in $M^{\#}$.

1. If $G(E)$ is a $C^{b}(M)$-module, consider $f \in C\left(M^{\#}\right)$ such that $0 \leq f \leq$ $1, f\left(\chi_{0}\right)=1$ and supp $f \subset V^{\#}\left(\chi_{0}, x_{1}, x_{2}, \ldots, x_{n}, \epsilon\right)$. Choose $x$ in $E$ so that $\chi_{0}(x)=1$ and take $y$ with $G(y)=G(x) f$. Then $\phi \neq V\left(\chi_{0}, y, \epsilon\right) \subset$ $V\left(\chi_{0}, x_{1}, x_{2}, \ldots, x_{n}, \epsilon\right)$ and $M$ is dense in $M^{\#}$.

2. If $E$ has an involution so that $\chi\left(x^{\star}\right)=\overline{\chi(x)}, \chi \in M, x \in \underline{E \text { and } E}$ has an identity, consider the element $x=\sum_{i=1}^{n}\left(x_{i}-\chi_{0}\left(x_{i}\right)\right)\left(x_{i}^{\star}-\overline{\chi_{0}\left(x_{i}^{\star}\right)}\right)$ of $E$. Then the set $V\left(\chi_{0}, x, \epsilon\right)$ is nonvoid and for every $\chi \in M$, we have:

$$
\begin{aligned}
\chi \in V\left(\chi_{0}, x, \epsilon\right) & \Longleftrightarrow\left|\chi(x)-\chi_{0}(x)\right|<\epsilon \\
& \Longleftrightarrow|\chi(x)|<\epsilon \\
& \Longleftrightarrow \Sigma_{i=1}^{n}\left|\chi\left(x_{i}\right)-\chi_{0}\left(x_{i}\right)\right|^{2}<\epsilon \\
& \Longrightarrow\left|\chi\left(x_{i}\right)-\chi_{0}\left(x_{i}\right)\right|<\epsilon \\
& \Longrightarrow \chi \in V\left(\chi_{0}, x_{1}, x_{2}, \ldots, x_{n}, \epsilon\right)
\end{aligned}
$$

This shows the density of $M$ in $M^{\#}$. 
Now, if $E$ has no identity, consider $x=\sum_{i=1}^{n} x_{i} x_{i}^{\star}-\chi_{0}\left(x_{i}\right) x_{i}^{\star}-\overline{\chi_{0}\left(x_{i}\right)} x_{i}$.

This is an element of $E$ and

$$
\begin{aligned}
\chi \in V\left(\chi_{0}, x, \epsilon\right) & \Longleftrightarrow \mid \Sigma_{i=1}^{n} \chi\left(x_{i}\right) \overline{\chi\left(x_{i}\right)}-\chi_{0}\left(x_{i}\right) \overline{\chi\left(x_{i}\right)}-\overline{\chi_{0}\left(x_{i}\right)} \chi\left(x_{i}\right)+ \\
& \quad+\Sigma_{i=1}^{n} \chi_{0}\left(x_{i}\right) \overline{\chi_{0}\left(x_{i}\right)} \mid<\epsilon \\
& \Longleftrightarrow \mid \Sigma_{i=1}^{n} \chi\left(x_{i}\right)\left[\overline{\chi\left(x_{i}\right)}-\overline{\chi_{0}\left(x_{i}\right)}\right]-\Sigma_{i=1}^{n} \chi_{0}\left(x_{i}\right)\left[\overline{\chi_{0}\left(x_{i}\right)}-\overline{\chi\left(x_{i}\right)} \mid<\epsilon\right. \\
& \Longleftrightarrow \mid \Sigma_{i=1}^{n}\left(\chi\left(x_{i}\right)-\chi_{0}\left(x_{i}\right)\right)\left(\overline{\chi\left(x_{i}\right)}-\overline{\chi_{0}\left(x_{i}\right)} \mid<\epsilon\right. \\
& \Longleftrightarrow \Sigma_{i=1}^{n}\left|\chi\left(x_{i}\right)-\chi_{0}\left(x_{i}\right)\right|^{2}<\epsilon \\
& \Longrightarrow\left|\chi\left(x_{i}\right)-\chi_{0}\left(x_{i}\right)\right|<\epsilon \\
& \Longrightarrow \chi \in V\left(\chi_{0}, x_{1}, x_{2}, \ldots, s_{n}, \epsilon\right) .
\end{aligned}
$$

Therefore $V\left(\chi_{0}, x, \epsilon\right) \subset V\left(\chi_{0}, x_{1}, x_{2}, \ldots, x_{n}, \epsilon\right)$ and $M$ is dense in $M^{\#}$.

3 . If $\mathbf{K}=\mathbf{R}$, and $x^{\star}=x$, the same proof as for 2 . holds.

Remark. 1. Since for a l.c.a. $E$ which is locally barrelled (i.e. every bounded set of $E$ is contained in a bounded disk $B$ so that $\left(E_{B},\|.\|_{B}\right)$ is barrelled), every weakly bounded subset of $E^{\prime}$ is strongly bounded [10], every character of such an algebra with property $(W)$ whose spectrum is metrisable is bounded in each of the cases 1.,2., and 3. above. As a special case, this holds if $E$ is a commutative complete locally m-convex algebra whose spectrum is metrisable.

2. With a similar proof as for 1. in Proposition 2.1, one can show that if $E$ is a regular commutative Hausdorff l.c.a. verifying (W), then $M$ is dense in $M^{\#}$. Here $E$ is said to be regular if for every $\chi \in M$ and every closed subset $F$ of $M$ with $\chi \notin F$, there is $x \in E$ such that $\chi(x)=1$ and that the restriction of $G(x)$ to $F$ is identically 0 .

Let $E$ be a commutative Hausdorff l.c.a. and $\widehat{E}$ its completion. A.Mallios [7] has shown that if $\widehat{E}$ is also a l.c.a. and if $M$ is locally 
equicontinuous, then $M=M_{\widehat{E}}$ holds topologically, where $M_{\widehat{E}}$ is the set $M$ equipped with the weak topology generated by $\widehat{E}$. If $F$ is al.c. space containing $E$ as a dense subspace, then for every $\chi \in M$, there is a continuous linear functional on $F$ the restriction of which to $E$ coincides with $\chi$. If we consider $M_{F}$, the set $M$ endowed with the weak topology generated by $F$ (notice that $E^{\prime}$ can be identified with $F^{\prime}$ ), then we are led to the question: When does the equality $M=M_{F}$ hold topologically?

With a similar proof as in [7], one can show

2. Proposition. In each of the following cases, the equality $M=$ $M_{F}$ holds topologically:

1. $M$ is a $k_{\mathrm{R}}$-space, and every compact subset of $M$ is equicontinuous.

2. $\left(C(M), \tau_{e}\right)$ is complete.

As consequence of this result, if $E$ is m-barrelled and $M$ a $k_{\mathbf{R}}$ space, then the equality above holds for every l.c. space $F$. In general, the equality does not hold even for strongly semisimple uniformly $A$ convex algebras. Take, for example, the algebra $E:=C[0,1]$ with the topology generated by the seminorms $\left(P_{B}\right)_{B}$, where $B$ is a finite subset of $[0,1]$ and

$$
P_{B}(f):=\max \left(\max _{x \in B}|f(x)|, \int_{0}^{1}|f(x)| d x\right), f \in C[0,1] .
$$

Here $M=[0,1]$ algebraically and topologically, but $M_{\widehat{E}}$ is the discrete space $[0,1]$.

Acknowledgements. This work was written while the author was preparing his dissertation at the university of Paderborn (Germany). He is indebted to D.A.A.D. for the support, to Professor K.D. Bierstedt for the facifities he provided him in Paderborn and to Professor M. Oudadess for his encouragements.

\section{References}


[1] Akkar, M.: Etude spectrale et structures d'algèbres topologiques ou bornologiques completes. Thèse sc. math. Bordeaux (1976).

[2] Allan, G.R.: A spectral theory for locally convex algebras. Proc. London Math. soc. 15 (1965), 399-421.

[3] Allan, G.R., Dales, H.G. and McClure, J.P.: Pseudo-Banach algebras. Studia Math. XL, (1971), 55-69.

[4] Cochran, A.C.: Representation of A-convex algebras. Proc. Amer. Math. Soc. 41 (1973), 473-479.

[5] Cochran, A.C.: Inductive limits of A-convex algebras. Proc. Amer. Math. Soc. 37 (1973), 489-496.

[6] Hogbe-Nlend, H.: Les fondements de la théorịe spectrale des algébres bornologiques. Bol. Soc. Brasil. Math. 3 (1973), 19-56.

[7] Mallios, A.: Topological algebras, selected topics. North-Holland Math. Studies 124 (1986)

[8] Michael, E.A.: Locally multiplicatively convex topological algebras. Memoirs Amer. Math. Soc. 11, Providence (1952).

[9] Morris, P.D. and Wulbert, D.E.: Functional representation of topological algebras. Pacific J. Math. 22 (1967), 323-337.

[10] Oubbi, L.: Counter-examples to some assertions of C. Bosh and J. Kucera. Publ. Math. Ecole Norm. Sup. Rabat 5 (1989), 39-43.

[11] Oudadess, M.: Théorèmes de structures et propriétés fondamentales des algébres localement uniformément $A$-convexes. C.R. Acad. Sc. Paris 296 (1983), 851-853.

[12] Prolla, J.B.: Bishop's generalized Stone-Weierstrass theorem for weighted spaces. Math. Ann. 191 (1971), 283-289.

[13] Warner, S.: Inductive limits of normed algebras. Trans. Amer. Math. Soc. 82 (1956), 190-216. 